\title{
Glutathione Reductase Activity in Serum and Liver Tissue of Human and Rat with Hepatic Damage
}

\author{
Hirokatsu Kumata, Kazuo Wakut, Hiroshi Suzuki, \\ Takehisa Sugawara, Insu Lim, Masao Otsuki, Tsuneo \\ Ozeki, Kiyomi Miura and Shoichi Yamagata \\ The Third Department of Internal Medicine, Tohoku University \\ School of Medicine, Sendai
}

\begin{abstract}
Kumata, H., Wakui, K., Suzuki, H., Sugawara, T., Lim, I., Otsuki, M., Ozeki, T. Mrura K. and Yamagata, S. Glutathione Reductase Activity in Serum and Liver Tissue of Human and Rat with Hepatic Damage. Tohoku J. exp. Med., 1975, $116(2), 127-132 \ldots$ Glutathione reductase activity of both serum and liver tissue homogenates was measured in normal controls and in cases of hepatic parenchymatous diseases, and the results were compared with those from animal experiments in which hepatic damage was produced by $\mathrm{CCl}_{4}$ injection. Glutathione reductase showed a different attitude from those of transaminases and alkaline phosphatases under these clinical and experimental conditions. Glutathione reductase activity increased in both serum and liver in patients with hepatic damage, and this increase occurred earlier than the changes in alkaline phosphatase activity. _— glutathione reductase; serum; liver homogenates; parenchymatous liver diseases
\end{abstract}

Since the report of a new chemical agent "glutathione" by Hopkins (1921), numerous efforts have been made to establish the biological importance of this substance. Glutathione plays an important role in the electron transport system in the liver, as pointed out by Hopkins and Elliott (1932). The reduction of glutathione takes place in the liver, and the enzyme which catalyzes this process is named glutathione reductase (Mann 1932). Meldrum and Tarr (1935) reported that in whole blood of the rat, TPNH works as the co-factor in the reduction of oxidized glutathione to the reduced form. The presence of glutathione in the liver was established by Rall and Lehninger (1952) and Racker (1955). Although the physiological significance of glutathione reductase in human sera has not well been understood, the determination of this enzyme was carried out in patients under various clinical conditions and it has been revealed that its activity increases in the initial phase of acute hepatitis as well as in malignant diseases with liver metastases (Manso and Wróblewsky 1958; Kerppola et al. 1959; West et al. 1961). By the assay for serum enzymes under various clinical conditions, we noticed a different behavior of this enzyme from those of other enzymes of hepatic origin including transaminases and alkaline phosphatase (unpublished observation). In regard to the mechanism of the elevation of reductase activity in liver diseases

Received for publication, December 25, 1974. 
West et al. (1961) proposed a theory that changes in permeability of the cell membranes may result in an increasing release of the enzyme into serum, as the reductase is a solubule, cytoplasmic enzyme. If it is the case, the reductase activity in serum should increase in proportion to the decreased activity in the liver. The purpose of the present study is to examine whether there is such a correlation between activities in serum and liver.

\section{Materials and Methods}

For the animal experiments, male adult rats of the Donryu strain weighing 180 to 220 $\mathrm{g}$ were used. The animals were divided into two groups, the control and the experimental. The control group received olive oil at a dose of $0.02 \mathrm{ml} / 100 \mathrm{~g}$ body weight, and the experimental group received an intraperitoneal injection of $10 \% \mathrm{CCl}_{4}$ dissolved in olive oil at the same dose. At $6,12,24$ and $36 \mathrm{hr}$ after injection, the animals were anesthetized with nembutal and sacrificed by blood aspiration through a cardiac puncture. Immediately, serum was separated, and liver homogenates were made by adding $0.25 \mathrm{M}$ sucrose solution and centrifuging for $10 \mathrm{~min}$ at $3000 \mathrm{rpm}$.

Twenty nine patients with various liver diseases were subjected to this study. They comprised 14 with acute hepatitis, 11 with chronic hepatitis, and 4 with liver cirrhosis. In addition, 4 apparently healthy subjects were examined as the controls. The diagnosis was made by clinical, laboratory and histological examinations. All patients received a laparoscopic examination when liver specimens were taken by liver biopsy. Liver homogenates were made as described above. Blood was taken at the fasting stage in the morning of the day of laparoscopic examination.

Glutathione reductase determination was done according to the method of Manso and Wróblewsky (1958). In the course of this study, it was found that glutathione reductase activity increased when blood was kept standing at room temperature for more than three days. In contrast, when serum was separated and allowed to stand at room temperature, a significant decrease was found in the enzyme activity after three days. But when serum was kept under $-20^{\circ} \mathrm{C}$, no significant decrease in activity progressed during a period of one month. Variation in incubation temperature from $20^{\circ} \mathrm{C}$ to $37^{\circ} \mathrm{C}$ for $20 \mathrm{~min}$ did not cause any significant changes in the results of determination. Incubation time is important, for different incubation times caused a considerable change in enzyme activity; the value was quadriplicated without incubation as compared with the value of the original $20 \mathrm{~min}$ incubation, doubled by $5 \mathrm{~min}$ incubation, and lowered to one third by $60 \mathrm{~min}$ incubation.

A slight decrease of the activity occurred when buffer solutions of $\mathrm{pH} 7.0$ and 8.8 were used. There was no difference in the activity between buffer solutions of $\mathrm{pH} 7.6$ and 8.1. Increase of TPNH concentration elevated the enzyme activity. Addition of an extra amount of oxidized glutathione, 0.01 to $0.05 \mathrm{mg}$, at the time of determination significantly elevated the activity of glutathione reductase.

All of these aspects influenced the determination, and the original procedure was strictly followed.

The activities of serum glutathione reductase (SGR) and liver glutathione reductase (LGR) were expressed as units/ml for SGR and units/mg wet liver tissue for LGR. GOT and GPT were measured by the Sigma-Frankel method. Serum GOT and GPT (SGOT, and SGPT) and liver GOT and GPT (LGOT and LGPT) activities were expressed in the same way. Alkaline phosphatase activity was measured by the Huggins and Talalay method modified by Yoshikawa and Hosoya (1949).

\section{Results}

In 4 apparently healthy subjects, LGR activity was found to be $6.0 \pm 2.0$ units which showed statistically significant correlation with LGOT and LGPT 

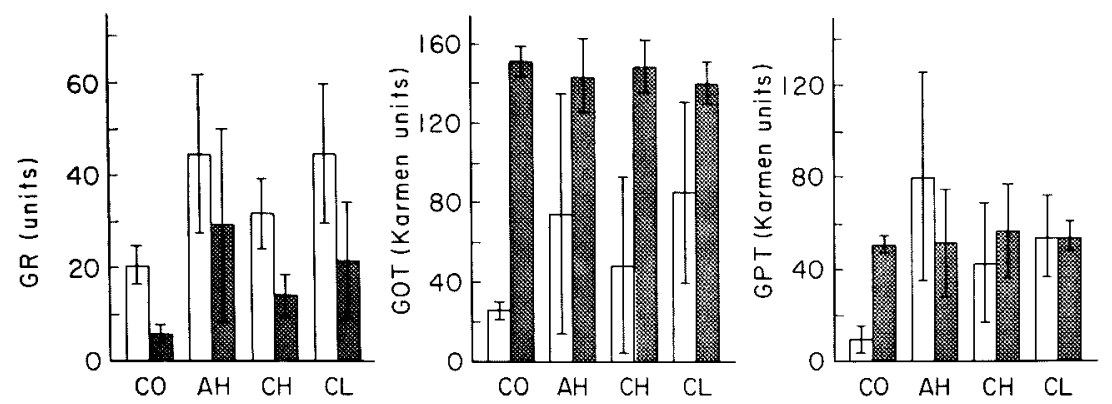

Fig. 1. Activities of GR, GOT and GPT in serum and liver homogenates of patients with liver diseases.
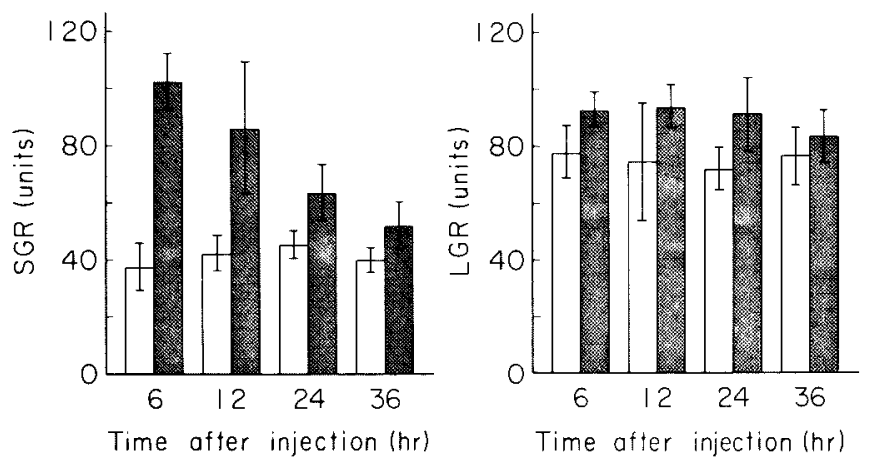

Fig. 2. Glutathione reductase in serum and liver homogenates of rats.

(corresponding $r$ and $p$ were $r=0.904$ and $0.943 ; p<0.01$ in both). The LGR was $29.6 \pm 21.1$ units in 14 patients with acute hepatitis, $13.5_{ \pm} 5.5$ units in 11 with chronic hepatitis and $22.0 \pm 12.5$ units in 4 with liver cirrhosis. Statistically significant correlation was found between LGR and LGOT in acute hepatitis $(r=$ $0.436, p<0.1)$, and LGR and LGPT in liver cirrhosis $(r=0.762, p<0.1)$ (Fig. 1).

In the control rats, the SGR activities at $6,12,24$ and $36 \mathrm{hr}$ after injection of olive oil were $38.0 \pm 8.7,42.8 \pm 6.4,46.5 \pm 5.1$ and $40.3 \pm 4.7$ units $/ \mathrm{ml}$, respectively. The corresponding values in the $\mathrm{CCl}_{4}$ treated rats were $102.8 \pm 10.1,86.4 \pm 23.4$, $64.0 \pm 10.1$ and $52.4 \pm 8.6$ units/ml (Fig. 2).

The LGR activities at $6,12,24$ and $36 \mathrm{hr}$ after injection in the control rats were $78.4 \pm 9.3,75.2 \pm 21.2,72.4 \pm 7.8$ and $76.8 \pm 10.4 \mathrm{units} / \mathrm{mg}$, and in the $\mathrm{CCl}_{4}$ treated rats the corresponding values were $93.6 \pm 6.0,94.8 \pm 8.2,92.0 \pm 13.4$ and $83.4 \pm 9.6$ units/mg (Fig. 2).

The serum and liver activities of GOT, GPT and alkaline phosphatase are shown in Figs. 3, 4 and 5. Significant correlation was found between LGR and LGOT activities at $6 \mathrm{hr}$ after $\mathrm{CCl}_{4}$ injection $(r=0.876, p<0.05)$.

\section{Discussion}

Glutathione reductase activity was found to be elevated in liver homogenates 

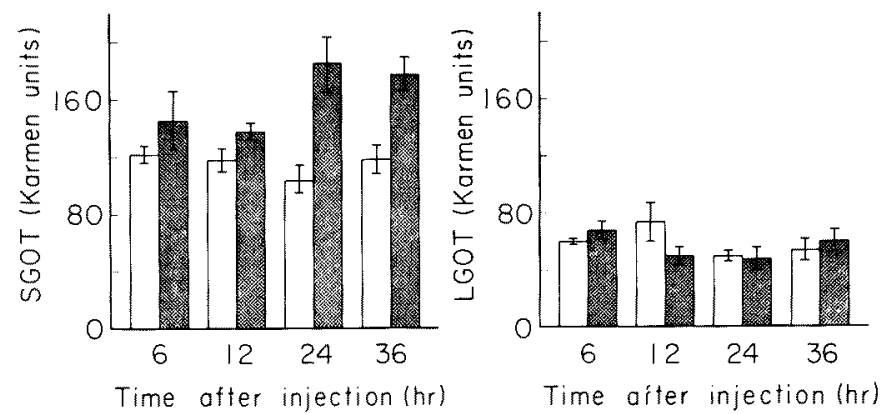

Fig. 3. GOT in serum and liver tissue homogenates of rats.

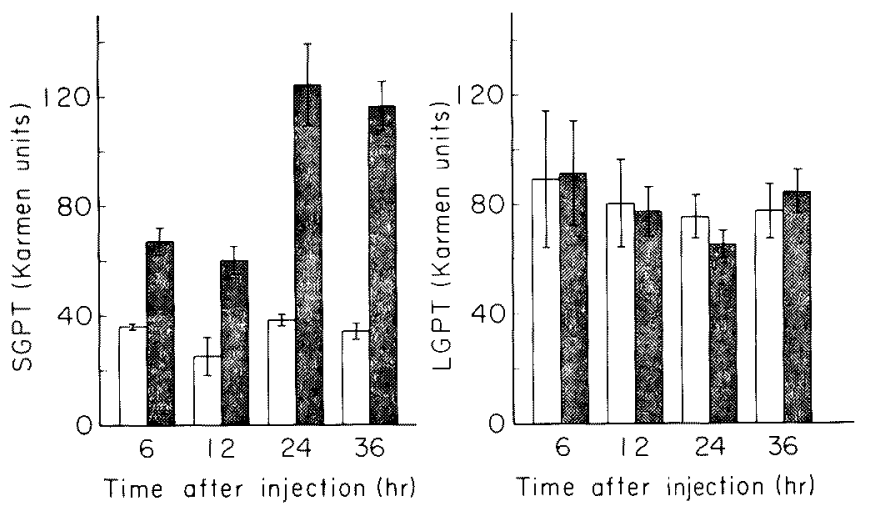

Fig. 4. GPT in serum and liver homogenates of rats.

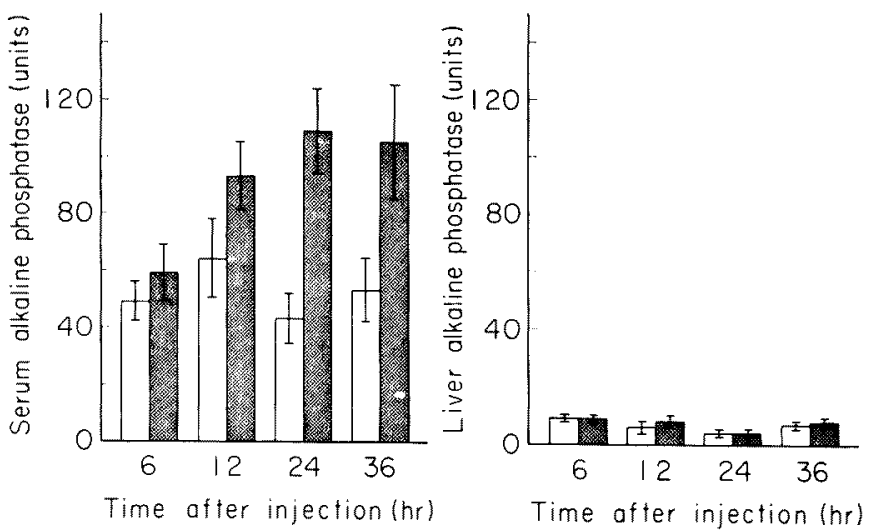

Fig. 5. Alkaline phosphatase in serum and liver homogenates of rats.

in diseased states as compared with the normal liver. The greatest rise was found in the acute hepatitis cases followed by liver cirrhosis and then chronic hepatitis. The normal values were close to those reported by Manso and Wróblewsky (1958). They noted that human hepatic tissue contained glutathione reductase in 
an amount of 3200 units/g wet tissue, but this value was the average of only two tissue determinations. Their material was taken at the time of autopsy within 5 hr after death. In our study, the specimens were taken at the time of laparoscopic examination using the needle biopsy technique. Nevertheless, the value reported by Manso and Wróblewsky (1958) well agrees to ours, that is, $6.0 \pm 2.0$ units/mg. When the LGR was compared with LGOT and LGPT, statistically significant correlation was found between LGR and LGOT in acute hepatitis and between LGR and LGPT in liver cirrhosis, but there was no parallelism between these enzymes in the other cases. It should be mentioned that activities of liver GOT and GPT in normal subjects in the present study were in the same magnitude as reported previously (Wróblewsky and LaDue 1956; Harada 1958; Kaito and Wakui 1965). The LGR increased invariably under diseased conditions, while either LGOT or LGPT did not always increase. In this regard, the attitude of LGR is similar to that of alkaline phosphatase (Kaito and Wakui 1965).

In animal experiments, the values for LGR corresponded fairly well to those reported by Bamji and Sharada (1972). It was found that SGR increased approximately three times the control level at initial $6 \mathrm{hr}$ after $\mathrm{CCl}_{4}$ injection. This initial increase lowered steadily, and at $36 \mathrm{hr}$ the activity almost returned to the control value. This rise of SGR was accompanied by the rise of LGR, though the LGR showed the peak at $12 \mathrm{hr}$. There is, thus, a parallelism between the serum and liver glutathione reductase activities under the experimental conditions. Both transaminases showed rises in the serum activity at 24 and $36 \mathrm{hr}$ and definite decreases in the liver activity at 12 to $24 \mathrm{hr}$. Alkaline phosphatase showed a gradual increase in serum, reaching the highest peak at $24 \mathrm{hr}$, but did not show any appreciable change in the liver. Thus, the behavior of glutathione reductase under the experimental conditions showed no parallelism with either one of these three enzymes. It should be also pointed out that the elevation of SGR occurred much earlier than the changes in SGOT and SGPT and that the decrease of SGR occurred again much earlier than the decreases in serum activities of both transaminases.

The tissue distribution of glutathione reductase shows a certain similarity to that of GPT (Manso and Wróblewsky 1958). The highest activity was found in the liver and kidney, followed by the pancreas, heart, thyroid, red cells and lungs in the descending order. On this basis, West et al. (1961) postulated the possibility that the SGR elevation should be due to the leakage or liberation of the enzyme from the liver. If it is so, one may expect a concomitant decrease in the activity in the liver. Certain enzymes such as GOT fall in this category (Harada 1958; Sakata 1960; Yamagata et al. 1962; Kaito and Wakui 1965). The present investigation, however, clearly demonstrated that the mechanism of the elevation of SGR in liver damage is different from that of the so-called "leaking enzymes," which are liberated from the liver in liver damage. (Takasugi 1961).

The process of liver damage induced by the administration of $\mathrm{CCl}_{4}$ can be divided into two phases; the initial early stage where massive glucose damping, 
arrest of bile secretion, sodium space expansion, etc. occur, and the later phase where secondary changes of liver tissue are caused by vascular changes (Bauer 1963). It is interesting to note that changes of glutathione reductase activities in serum and liver occur in the early stage, which is difficult to explain on the basis of enzyme induction. Possibility of the decreased up-take and catabolism of the enzyme may explain the phenomena.

\section{References}

1) Bamji, M.S. \& Sharada, D. (1972) Hepatic glutathione reductase and riboflavin concentrations in experimental deficiency of thiamin and riboflavin in rats. $J$. Nutr., 102, 443-448.

2) Bauer, P.W. (1963) Liver circulation and function. Physiol. Rev., 43, 115-213.

3) Harada, T. (1958) Studies on serum transaminase in liver diseases. J.J.G.E. (Jap.), $55,591-604$.

4) Hopkins, F.G. (1921) On an autoxidisable constitutent of the cell. Biochem. J., 15, 285-305.

5) Hopkins, F.G. \& Elliott, K.A.C. (1932) The reduction of glutathione to cell respiration with special reference to hepatic tissue. Proc. roy. Soc., B., 109, 58-88.

6) Kaito, I. \& Wakui, K. (1965) The significance of the determination of hepatic enzymes in various clinical conditions. Tohoku J. exp. Med., 86, 23-32.

7) Kerppola, W., Nikkila, E.A. \& Pitkanen, E. (1959) Serum TPN-linked enzymes, glucose-6-phosphate dehydrogenase, isocitric dehydrogenase and glutathione reductase activities in health and in various disease states. Acta med. scand., 164, 357-365.

8) Mann, P.J.G. (1932) The reduction of glutathione by a liver system. Biochem. J., 26, 785-790.

9) Manso, C. \& Wróblewsky, F. (1958) Glutathione reductase activity in blood and body fluids. J. clin. Invest., 37, 214-218.

10) Meldrum, N.U. \& Tarr, H.L.A. (1935) The reduction of glutathione by the WarburgChristian system. Biochem. J., 29, 108-115.

11) Racker, E. (1955) Glutathione reductase from baker's yeast and beef liver. J. biol. Chem., 217, 855-865.

12) Rall, T.W. \& Lehninger, A.L. (1952) Glutathione reductase of animal tissues. $J$. biol. Chem., 194, 119-130, 1952.

13) Sakata, Y. (1960) Studies on enzymatic activities in serum and liver of patients with liver diseases. J.J.G.E. (Jap.), 57, 121-141.

14) Takasugi, T. (1961) Diagnosis of liver diseases. Nippon Naikagakkai Zasshi (Jap.), 50, 527-551.

15) West, M., Berger, C., Rony, H. \& Zimmerman, H.J. (1961) Glutathione reductase in sera of normal subjects and of patients with various diseases. J. Lab. clin. Med., $57,946-954$.

16) Wróblewsky, F. \& LaDue, J.S. (1956) Serum glutamic pyruvic transaminase (SGP-T) in heaptic disease: A preliminary report. Ann. intern. Med., 45, 801-811.

17) Yamagata, S., Wakui, K. \& Uchimi, M. (1962) Liver enzymes: pathophysiological significance. Sogo Rinsho (Jap.), 11, 1606-1614.

18) Yoshikawa, H. \& Hosoya, N. (1949) Determination of alkaline phosphatase using phenolphthalein-phosphoric-ester as substrate. Kosokagaku Symposium. (Jap.), 2, $54-60$. 